\title{
Unter NOAK nicht höher als unter VKA
}

Fragestellung: Unterscheiden sich die einzelnen nicht VitaminK-abhängigen oralen Antikoagulanzien (NOAK) hinsichtlich des Risikos gastrointestinaler Blutungen?

Hintergrund: Die NOAK wurden zur Schlaganfallprävention bei Patienten mit Vorhofflimmern und zur Prophylaxe und Therapie tiefer Beinvenenthrombosen und Lungenembolien entwickelt. Das Verschreibungsverhalten von Neurologen ist geprägt durch den Wunsch, Schlaganfälle und insbesondere intrakranielle Blutungen zu verhindern. Hausärzte, Internisten und Gastroenterologen sind eher dar-

Burr N, Lummis K, Sood R et al. Risk of gastrointestinal bleeding with direct oral anticoagulants: a systematic review and network meta-analysis. Lancet Gastroenterol Hepatol 2017; 2: $85-93$ an interessiert, systemische, insbesondere gastrointestinale Blutungen $\mathrm{zu}$ vermeiden. Daher ist es wichtig, das Risiko gastrointestinaler Blutungen der NOAK mit Heparinen und Vitamin-K-Antagonisten (VKA) zu vergleichen.
Patienten und Methodik: Die Autoren führten eine systematische Literaturrecherche durch. Sie identifizierten 31 Studien mit insgesamt 287.692 Patienten mit einer kumulativen Antikoagulationszeit von 230.090 Jahren. Der primäre Endpunkt der Analyse waren schwerwiegende gastrointestinale Blutungen, der sekundäre Endpunkt alle gastrointestinalen Blutungen.

Ergebnisse: Das Risiko schwerwiegender gastrointestinaler Blutungen war mit den NOAK vergleichbar mit dem von Warfarin und niedermolekularem Heparin (Faktor-Xa-Hemmer vs. Warfarin 0,78, Warfarin vs. Dabigatran 0,88, Faktor-Xa-Hemmer vs. niedermolekulares Heparin 1,02, niedermolekulares Heparin vs. Dabigatran 0,67). Für den sekundären Endpunkt „alle gastrointestinalen" Blutungen hatten die Faktor-Xa-Hemmer ein geringeres Risiko als Warfarin und Dabigatran.

Schlussfolgerungen: Unter NOAK ist das Risiko gastrointestinaler Blutungen vergleichbar wie bei der Behandlung mit Warfarin oder niedermolekularem Heparin.

\section{Die Schlaganfallstudien zeichnen ein differenzierteres Bild}

Diese Metaanalyse belegt, dass über alle NOAK hinweg das Risiko schwerwiegender gastrointestinaler Blutungen für die NOAK nicht höher ist als unter Warfarin. Für die Schlaganfallprävention bei Patienten mit Vorhofflimmern gilt dies allerdings nicht uneingeschränkt. So ist das Risiko gastrointestinaler Blutungen verglichen mit Warfarin erhöht für die hohe
Dosis von Edoxaban, die hohe Dosis von Dabigatran und Rivaroxaban. Das Blutungsrisiko ist vergleichbar für die niedrige Dosis von Dabigatran und reduziert für Apixaban. Diese Aspekte aus den großen randomisierten Studien zur Schlaganfallprävention bei Vorhofflimmern wurden leider in der vorliegenden Metaanalyse nicht berücksichtigt.

\section{Segen oder Fluch?}

Fragestellung: Untersucht wurden die Effekte von Cannabiskonsum und -substanzstörungen auf Verlauf und Outcome einer schweren Depression über eine 3-Jahres-Periode.

Hintergrund: Cannabis stellt nach Tabak und Alkohol die am weitesten verbreitete Droge weltweit dar. Besonderes unter Patienten mit psychiatrischen Erkrankungen zeigt sich eine hohe Prävalenz von Cannabiskonsumenten. Trotz des bekannten negativen Einflusses von Cannabiskonsum auf verschiedene psychiatrische Erkrankungen, insbesondere psychotischer Störungen, kommt die aktuelle Literatur hier zu widersprüchlichen Ergebnissen. Wie sich Cannabiskonsum tatsächlich auf Verlauf und Outcome einer Depression auswirkt, ist bislang unklar.
Patienten und Methodik: In der vorliegenden Studie wurden die Daten der US-amerikanischen Studie „National Epidemiologic survey on Alcohol and Related Conditions“ (NESARC), eine nationale repräsentative Studie, initiiert durch das „National Institute of Alcohol Abuse and Alcoholism“, verwendet. Für diese longitudinale Umfrage unter nicht institutionalisierten in den USA lebenden über 18 Jahre alten Erwachsenen wurden zu zwei Zeitpunkten Probanden rekrutiert: bei der sogenannten „Wave 1“ von 2001 bis 2002 mit Einschluss von 43.093 Teilnehmern und „wave 2“ von 2003 bis 2004 mit 34.653 Probanden. Das heißt, 86,7 \% konnten beim 3-Jahres-Follow-up erneut eingeschlossen werden. Die Teilnehmer der aktuellen Studie stellten eine Subgruppe dar, mit der Voraussetzung der Diagnose einer schweren Depression im Jahr zuvor (Definition: mindes- 